\title{
la dilatance des roches
}

\author{
P. SIRIEYS \\ Laboratoire de tectonophysique — U.P.S.-I.N.S.A. Toulouse
}

\section{INTRODUCTION}

La microfissuration des roches se manifeste par un accroissement de volume du milieu, la dilatance. Le comportement dilatant est une des caractéristiques essentielles des massifs rocheux, il se manifeste dans de nombreux cas (naturels ou provoqués) lors de variations de l'état de contrainte naturel.

\section{LA DÉFORMATION DILATANTE}

Le comportement dilatant est une caractéristique des milieux rocheux fissurés et/ou fissurables. Il caractérise la déformation du milieu sous l'effet de création de fissures qui se superposent éventuellement à une fissuration antérieure. La dilatance des roches fragiles, déjà mise en évidence par Brace et al. (1966) se manifeste par une augmentation de volume du milieu, qui résulte d'une dérive de la loi de comportement par rapport à la loi linéaire élastique. La déformation s'exprime par la relation:

$$
\underline{\epsilon}=\underline{e}+\underline{y}
$$

en notant respectivement $\underline{\epsilon}$, $\underline{e}$ et $\gamma$ les tenseurs petite déformation totale, élastique et inélastique. La déformation $\gamma$ est appelée déformation dilatante, car son premier invariant $\gamma_{i j}$, noté $\theta$, est positif (sous la convention des extensions positives), elle caractérise la fissuration induite au cours de la petite déformation.

La dilatance est caractérisée par le scalaire $\theta$ (dilatation cubique). Cependant la déformation dilatante ne se réduit pas à sa partie isotrope, elle comporte une partie déviatoire qui est liée à celle des efforts, c'est-à-dire à celle de $\underline{e}$, et à la structure (anisotropie du milieu). Ainsi, pour une roche fissurable $\gamma$ induit une structure, dite structure de fissuration, et un comportement anisotrope, pour un milieu déjà fissuré $\gamma$ modifie l'anisotropie de la structure de fissuration initiale.

\section{LE SEUIL}

La déformation dilatante apparaît généralement seulement à partir d'un seuil de contrainte, pour lequel la déformation cesse d'être strictement élastique. Ce seuil, le seuil de dilatance, est caractérisé, pour une roche pseudo-isotrope, par une relation $f\left(\sigma_{1}, \sigma_{2}, \sigma_{3}\right)=0$ entre les trois contraintes principales $\sigma_{1}, \sigma_{2}, \sigma_{3}$, appelé critère de dilatance, il est imagé dans l'espace des contraintes $E_{3}$ par une surface $S_{d}$ (la surface limite de dilatance) intérieure à la surface $S_{\text {, }}$ (surface limite de rupture fragile). La surface $S_{d}$ (fig. 1) a été mise en évidence expérimentalement, notamment par Saint-Leu et al. (1973), Cherry et al. (1975). Sa méridienne, dans $E_{3}$, est assimilable à une droite, le critère de dilatance peut donc être caractérisé par un critère de Drucker-Prager de la forme:

$$
\mathrm{J}_{2}^{\prime+1}-\alpha \mathrm{I}_{1}-\mathrm{K}=0
$$

où $I_{1}$ et $J_{2}$ sont respectivement le premier invariant de l'état de contrainte $\underline{g}$ et le second invariant principal de o', déviateur de o. La surface $S_{d}$ est alors représentée par un cône dé révolution. A titre d'exemple, en compression monoaxiale, le seuil de dilatance du granite du Sidobre est de l'ordre de $30 \%$ du seuil de rupture, celui du marbre de Carrare est sensiblement nul. 


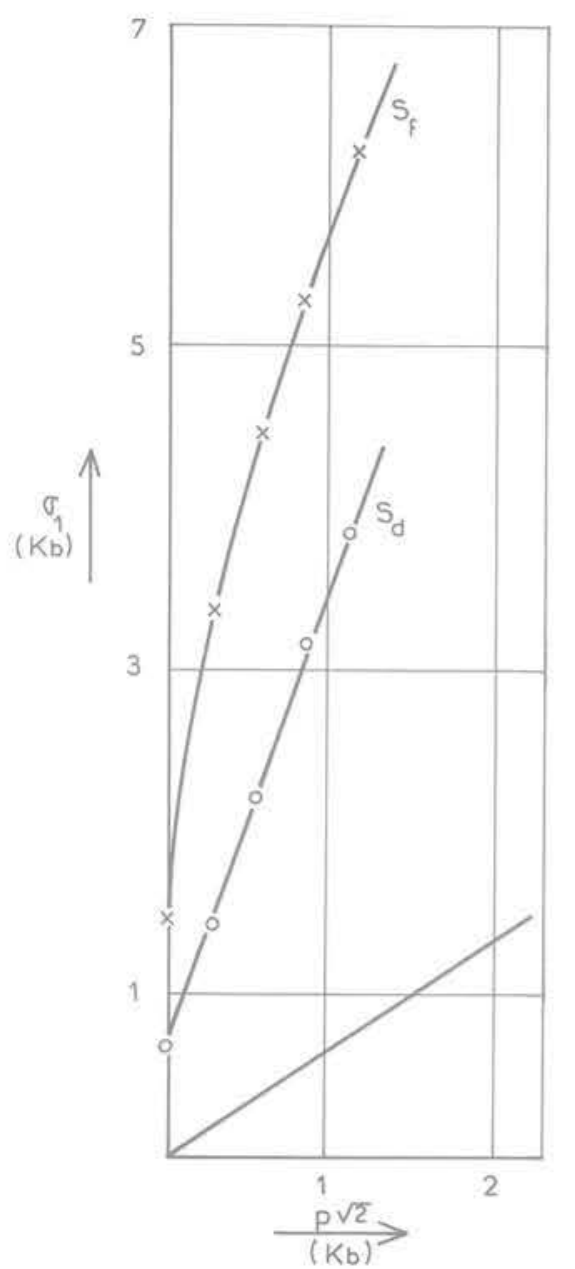

Fig. 1. - Le seuil de dilatance - Loi f $\left(\sigma_{1}, \sigma_{2}, \sigma_{3}\right)=0$ (Granite du Sidobre, d'après Saint-Leu et al., 1973) $S_{d}=$ surface de dilatance, $S_{f}=$ surface

de fracturation, $p=$ pression de confinement.

Dans $E_{3}$, l'espace entre $S_{d}$ et $S_{f}$, est appelé espace dilatant; la forme des surfaces $S_{d}$ et $S_{f}$ montre que la dilatance s'atténue lorsque la pression de confinement croît.

\section{LA LOI DE DILATANCE}

Le seuil $S_{d}$ étant atteint, la loi reliant la variation de volume inélastique aux contraintes s'exprime par une relation (Nur, 1975) entre invariants de la forme:

$$
\theta=\delta I_{2}^{n / 2}
$$

$\left(\mathrm{I}_{2}\right.$ est le second invariant de $\left.\underline{\mathrm{g}}\right)$. Elle fait intervenir un coefficient $n$ de l'ordre de 2 qui caractérise la dilatance due à la fissuration et un coefficient $\delta$ caractéristique de la roche et de son état fissural (fig. 2). Cette loi, de type parabolique, est notamment bien vérifiée lors de tests monoaxiaux, pour le granite du Sidobre et le marbre de Carrare (Saint-Leu et al., op. cit.).

\section{5. ÉVOLUTION DE LA DILATANCE, ENDOMMAGEMENT}

La fissuration induite, lorsque le seuil $\mathrm{S}_{\mathrm{d}}$ est atteint et dépassé, entraîne un endommagement de la roche initialement saine, ses qualités mécaniques sont affai-

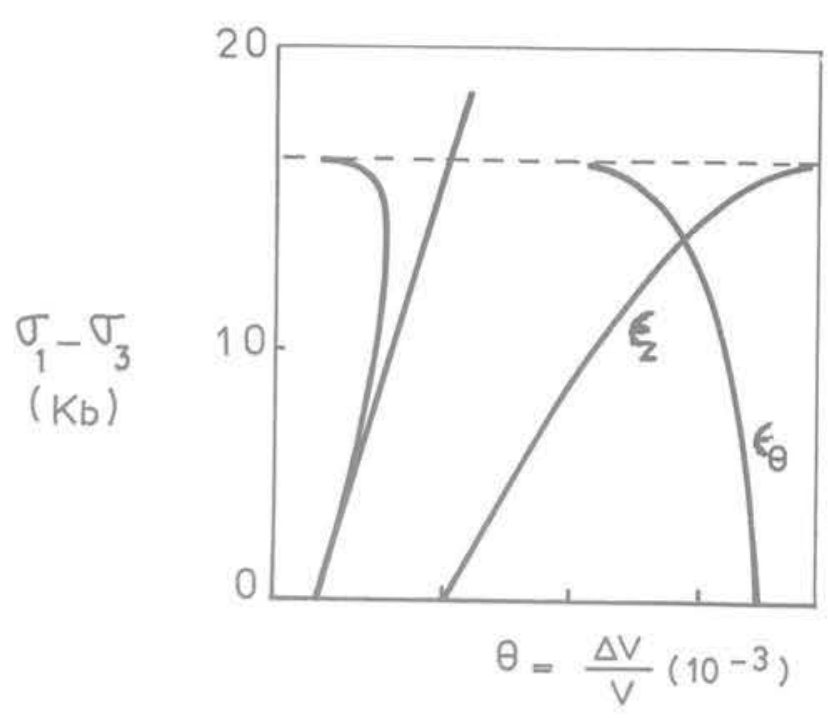

Fig. 2. - La loi de dilatance. Variation de volume $\theta$ en fonction de $\left(\sigma_{1}-\sigma_{3}\right)$

(Granite de Westerley, d'après Brace et al., in Nur, 1974).

blies. Alors qu'un trajet de chargement intérieur à $S_{d}$ n'altère pas les constantes mécaniques de la roche (module, résistance), la pénétration dans l'espace dilatant les affaiblit. La question se pose alors de l'évolution de la dilatance sous chargements ultérieurs.

Sous cycles de charge-décharge coaxiaux (de même orientation) dont le maximum en contraintes est inférieur à un seuil intérieur au domaine dilatant

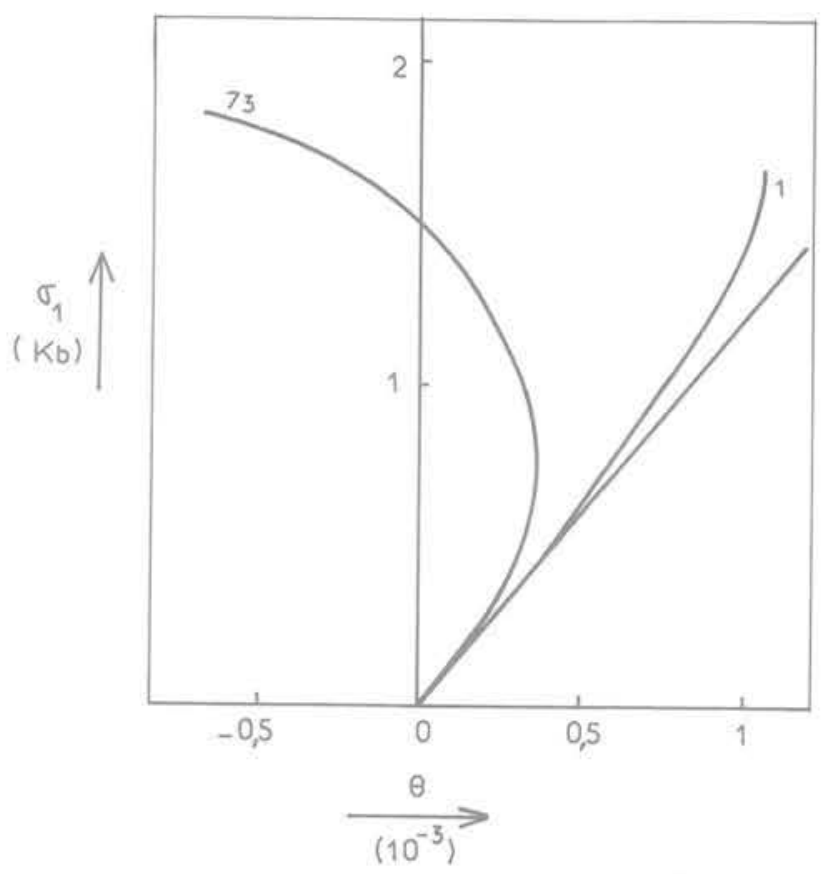

Fig. 3. - Evolution de la dilatance sous charges cycliques. Essais monoaxiaux.

(Granite du Sidobre, d'après Saint-Leu et al., 1971). $\sigma_{1}=$ contrainte monoaxiale,

$\theta=$ variation de volume, cycles $n^{\circ} 1$ et $n^{\circ} 73$ 
(Saint-Leu et al., 1971), la déformation inélastique est réversible et les diagrammes effort-déformation se superposent; $\theta$ n'évolue pas d'un cycle à l'autre, la fissuration est stable (on dit parfois que les fissures « respirent»). Par contre sous cycles coaxiaux dont le maximum dépasse ce seuil, les résultats expérimentaux (fig. 3) montrent que le seuil de dilatance n'est pas modifié à chaque cycle, que la loi de dilatance [3] conserve une allure parabolique ( $\mathrm{n}$ voisin de 2) mais avec un coefficient $\delta$ croissant à chaque cycle. II en résulte un endommagement progressif de la roche, la fissuration évoluant à chaque cycle.

\section{ANISOTROPIE}

Il convient de distinguer entre l'anisotropie de la déformation dilatante des roches (cf. §2) et la dilatance des roches anisotropes.

Pour les roches anisotropes, le mécanisme de dilatance (seuil et loi reliant $\gamma$ à $\sigma$ ) est anisotrope (Hadley, 1975). Le seuil, défini pār [2], fait alors intervenir l'orientation $(\alpha, \beta, \gamma)$ de $\underline{\sigma}$ par rapport à la structure, et s'écrit sous la forme $\mathrm{f}\left(\sigma_{1}, \sigma_{2}, \sigma_{3}, \alpha, \beta, \gamma\right)=0$. Les résultats expérimentaux sur roches schisteuses (Lerau et al., 1981) montrent que la dilatance n'apparaît que pour une plage d'orientation de $\sigma$ par rapport à la structure (fig. 4). Des compressions triaxiales de révolution, permettent de définir le cône de dilatance, tel que seule la direction principale majeure de $\sigma$ intérieure à ce cône permet l'apparition de la dilatance. Le seuil

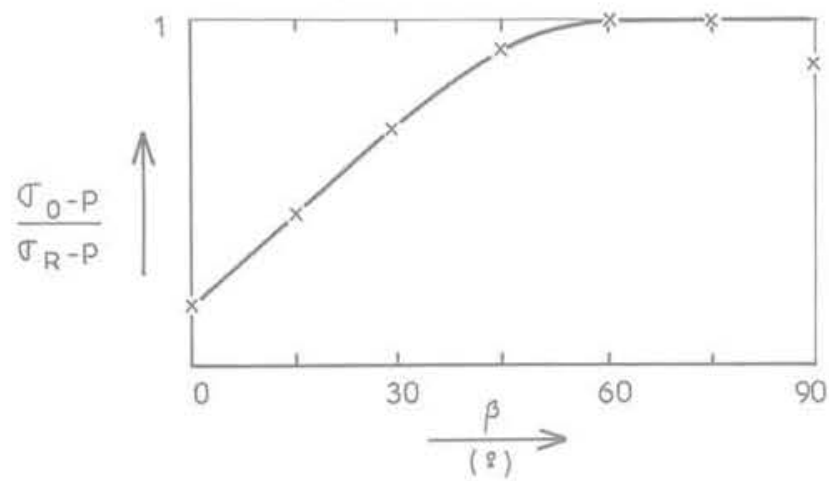

Fig. 4. - Dilatance des roches anisotropes. Plage dilatante.

(Schistes de Lacaune, d'après Lerau et al., 1981). $\sigma_{0}=$ seuil de dilatance, $\sigma_{R}=$ résistance à la rupture, $p=$ pression de confinement

$\beta=$ angle de $\sigma$, avec la normale à la schistosité. varie donc avec l'orientation des efforts, dans la plage dilatante, la loi de dilatance étant toujours sensiblement du second ordre $(n=2)$.

\section{CONCLUSION}

Finalement la dilatance est la manifestation du point de vue des déformations, de la création (ou du développement) de fissures intra (ou inter) cristallines qui se noient aux joints de grains ou dans la fissuration antérieure. $\mathrm{Ce}$ phénomène est également observable lors de tests acoustiques.

Pour une roche pseudo-isotrope la déformation dilatante induit une structure fissurale anisotrope (orientée par le tenseur des déformations élastiques). Pour une roche anisotrope, déjà fissurée, la nouvelle fissuration se superpose à l'ancienne par mécanisme de bifurcation de fissures; la fracturation du milieu s'effectue alors selon des surfaces composées d'éléments appartenant à chaque réseau fissural (ruptures mixtes)

\section{BIBLIOGRAPHIE}

BRACE, W.F.; PAULDING, B.W.; SCHOLZ, C. (1966). - Dilatancy in the fracture of cristalline rock - J. Geophys. Res., 77, p. 3939-3953.

CHERRY, J.T.; SCHOCK, R.N.; SWET, J. (1975). A theoretical model of the dilatant behaviour of a brittle rock - Pure and applied Geophysics, 113, p. $183-186$.

HADLEY, K. (1975). - Azimuthal variation of dilatancy - J. Geophys. Res., 80, n³5, p. 4835-4850.

LERAU, J.; SAINT-LEU, C.; SIRIEYS, P. (1981). Anisotropie de la dilatance - Rock mechanics $n^{\circ} 13$, Springer Verlag, Wien, p. 185-196.

NUR, A. (1974). - Tectonophysique: l'étude des relations entre les déformations et les forces agissant à l'intérieur du globe terrestre - C.R. $3^{e}$ Cong. S.I.M.R.

NUR, A. (1975). - A note on the constitutive law for dilatancy - Pure and applied Geophysics, 113, p. 197-206.

SAINT-LEU, C.; SIRIEYS, P. (1973). - Inélasticité et rupture du granite sous contraintes triaxiales - C.R. Acad. Sc. Paris, t. 276, série A, p. 817-820.

SAINT-LEU, C. ; SIRIEYS, P. (1971). - La fatigue des roches - C.R. Symp. S.M.I.R. sur la fissuration des roches. Nancy, n० II-18. 
